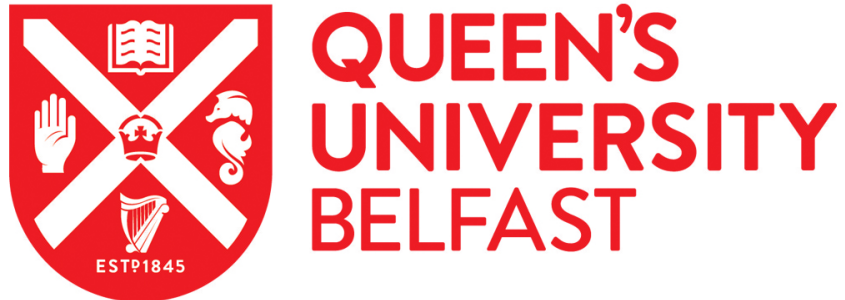

\section{The complex angular momentum (CAM) route to reactive scattering resonances: from a simple model to the $\mathrm{F}+\mathrm{H}-2->\mathrm{HF}+\mathrm{H}$ reaction}

Sokolovski, D. (2008). The complex angular momentum (CAM) route to reactive scattering resonances: from a simple model to the $F+H-2->H F+H$ reaction. 058118-1-058118-9. Paper presented at Meeting on Molecular and Nanodynamics - From Atoms to Biomolecules, Rome, Italy. https://doi.org/10.1088/0031-8949/78/05/058118

Queen's University Belfast - Research Portal:

Link to publication record in Queen's University Belfast Research Portal

\section{General rights}

Copyright for the publications made accessible via the Queen's University Belfast Research Portal is retained by the author(s) and / or other copyright owners and it is a condition of accessing these publications that users recognise and abide by the legal requirements associated with these rights.

Take down policy

The Research Portal is Queen's institutional repository that provides access to Queen's research output. Every effort has been made to ensure that content in the Research Portal does not infringe any person's rights, or applicable UK laws. If you discover content in the Research Portal that you believe breaches copyright or violates any law, please contact openaccess@qub.ac.uk. 
The complex angular momentum (CAM) route to reactive scattering resonances: from a simple model to the $\mathrm{F}+\mathrm{H}_{2} \rightarrow \mathrm{HF}+\mathrm{H}$ reaction

This article has been downloaded from IOPscience. Please scroll down to see the full text article. 2008 Phys. Scr. 78058118

(http://iopscience.iop.org/1402-4896/78/5/058118)

View the table of contents for this issue, or go to the journal homepage for more

Download details:

IP Address: 158.227.18.153

The article was downloaded on 22/02/2011 at 18:05

Please note that terms and conditions apply. 


\title{
The complex angular momentum (CAM) route to reactive scattering resonances: from a simple model to the $\mathrm{F}+\mathrm{H}_{2} \rightarrow \mathrm{HF}+\mathrm{H}$ reaction
}

\author{
D Sokolovski \\ E-mail: d.sokolovski@qub.ac.uk \\ Received 10 September 2008 \\ Accepted for publication 15 September 2008 \\ Published 5 November 2008 \\ Online at stacks.iop.org/PhysScr/78/058118
}

School of Mathematics and Physics, Queen's University of Belfast, BT7 1NN, UK

\begin{abstract}
We advocate the use of the complex angular momentum (CAM) approach for quantitative analysis of the resonance effects in reactive differential and integral cross sections. We start by reviewing a simple potential scattering model and progress to analyse the $\mathrm{F}+\mathrm{H}_{2}(v=0, j=0) \rightarrow \mathrm{HF}\left(v^{\prime}=2, j^{\prime}=0\right)+\mathrm{H}$ reaction recently considered by Cavalli and De Fazio (2007 Phys. Scr. 76 C21) within the conventional complex energy plane approach. We argue that although the two approaches have the same origin, preference may be given to the CAM method whenever a quantity of interest is obtained by summing a partial wave series.
\end{abstract}

PACS numbers: 82.20.Ej, 82.20.Fd, 82.20.Ln

(Some figures in this article are in colour only in the electronic version.)

\section{Introduction}

The presence and role of resonances in chemical reaction dynamics continues to be the subject of intense discussions in the literature (see, for example, [1]). To identify resonances one may examine the behaviour of the Smith's time-delay matrix [2], essentially a matrix of energy derivatives of the phases of the $S$-matrix elements, sensitive to the presence of complex energy (CE) poles close to the real energy axis. Alternatively, one may look for a signature a resonance or resonances produce in observable quantities. Differential cross sections (DCS) are good candidates for such a search: even if a resonance is short-lived a rapidly rotating metastable complex formed by the collision partners may break up into angular regions not reached by direct trajectories and significantly change the shape of the angular distribution. State-to-state integral cross sections (ICS) may be less sensitive to the capture in metastable states but are easier to measure and, for this reason, also important. Recently, Cavalli and De Fazio [3] presented a time-delay study of the resonance effects in the $\mathrm{F}+\mathrm{H}_{2} \rightarrow \mathrm{HF}+\mathrm{H}$ reaction caused by rotational alignment of the transition state and the exit channel van der Waals resonances. In this paper, we will provide a complementary view of the same problem.

While the authors of [3] looked for the positions of the CE poles $E_{n}(J)$ at real (integer) values of the total angular momentum $J$, we will study the behaviour of the complex angular momentum (CAM) Regge poles $J_{n}(E)$ considered for various real values of the energy $E$. It is important to stress that the two approaches have a common origin: there exists [4] a complex valued function $\mathcal{J}(E)$ (single-valued on a multi-sheet Riemann surface $\mathcal{R}$ ) such that inserting in the Schrödinger equation a (complex or real) energy $E$ and the angular momentum $\mathcal{J}(E)$ results in a solution which is regular at the origin and contains only outgoing waves in the far asymptotic region. Thus, tracing the value of $\mathcal{J}(E)$ along the real $E$-axis on the $n$th Riemann sheet of $\mathcal{R}$ gives the Regge trajectory $J_{n}(E)$. Conversely, choosing on the $n$th Riemann sheet a path such that on it $\mathcal{J}(E)$ is real, following the path until $\mathcal{J}$ reaches an integer value $m$ and taking down the corresponding complex value of the energy, yields the position of the $n$th CE pole, $E_{n}^{J=m}$, for real integer $J=m$. Equivalently, the same $\mathrm{CE}$ pole position can be found by evaluating the inverse of $\mathcal{J}(E), \mathcal{E}(J)$, and then taking down 
its value at an integer $J=m$ on the corresponding Riemann sheet of its own Riemann surface $\mathcal{R}^{\prime}$. This simple observation demonstrates that both types of pole positions can be derived from the same analytical function $\mathcal{J}(E)$ which can be obtained, at least in principle, by solving the corresponding Schrödinger equation.

With the CAM and CE poles so closely related, why should the preference be given to one type of poles and not to the other? The reason is that the observables of interest, such as DCS and ICS, are given by partial wave sums (PWS) over the (integer) values of the angular momentum rather than the energy, whose value is fixed. Such sums can be converted into integrals, and it is evaluation of integrals for which the poles are best suited. Thus, with the integration performed over $J$ and not $E$, Regge poles are likely to be more useful for quantifying resonance effects in both DCS and ICS than their CE counterparts. The CE poles are, on the other hand, far better known than the Regge poles (for the most recent review of the CAM poles applications in molecular collisions see [5]), and the purpose of this paper is to demonstrate and advertise the usefulness of the CAM approach to a, perhaps, sceptical reader. With this in mind, we will concentrate on the results obtained in analysing both the DCS and the ICS, and refer to our earlier publications whenever mathematical rigour is required. The rest of the paper is organized as follows. In section 2, we discuss the Regge trajectories, DCS and ICS for a simple potential model which, despite its simplicity, captures the main properties of realistic reactive systems modelled by state-of-the art computer simulations. In section 3, we present a CAM analysis of the title reaction and stressing its similarity with that of section 2 . Section 4 contains our conclusions.

\section{A simple (yet helpful) model}

We begin our analysis of the effects a quantum mechanical resonance may have on such observables as reactive DCS and ICS by analysing a simple potential model already used for similar purposes, for example, in [6]. The model consists of a hard-sphere core of radius $R^{\prime}$ surrounded by a thin semi-penetrable concentric layer of radius $R=R^{\prime}+d, d \ll R$ which we will represent by a potential $\delta$-function (figure 1 ). Thus the effective potential encountered by the scattered particle of unit mass and angular momentum $J$ is

$$
\begin{aligned}
V(r)= & \Omega \delta\left(R^{\prime}+d\right)+J(J+1) / 2 r^{2} \text { for } r>R^{\prime}, \\
& \text { and } \infty \text { otherwise. }
\end{aligned}
$$

Semiclassical conditions usually hold for atomic and molecular collisions, and in the following we will also assume that the de Broglie wavelength of the particle is much smaller than the size of the scatterer $k R \gg 1$, where $k \equiv(2 E)^{1 / 2}$ and $E$ is the particle's energy. We can, therefore, describe scattering in terms of rectilinear classical trajectories (rays) which, if $\Omega$ is large, mostly reflect off the outer potential shell. Not all trajectories will, however, be reflected as the radial potential (1) supports metastable potential levels trapped in the well between the core and the outer shell. For $J=0$, the position of the lowest level is given by $E_{0} \approx \pi^{2} / 2 d^{2}$, while its width $\Gamma_{0}$ is determined by the barrier strength $\Omega$. A particle

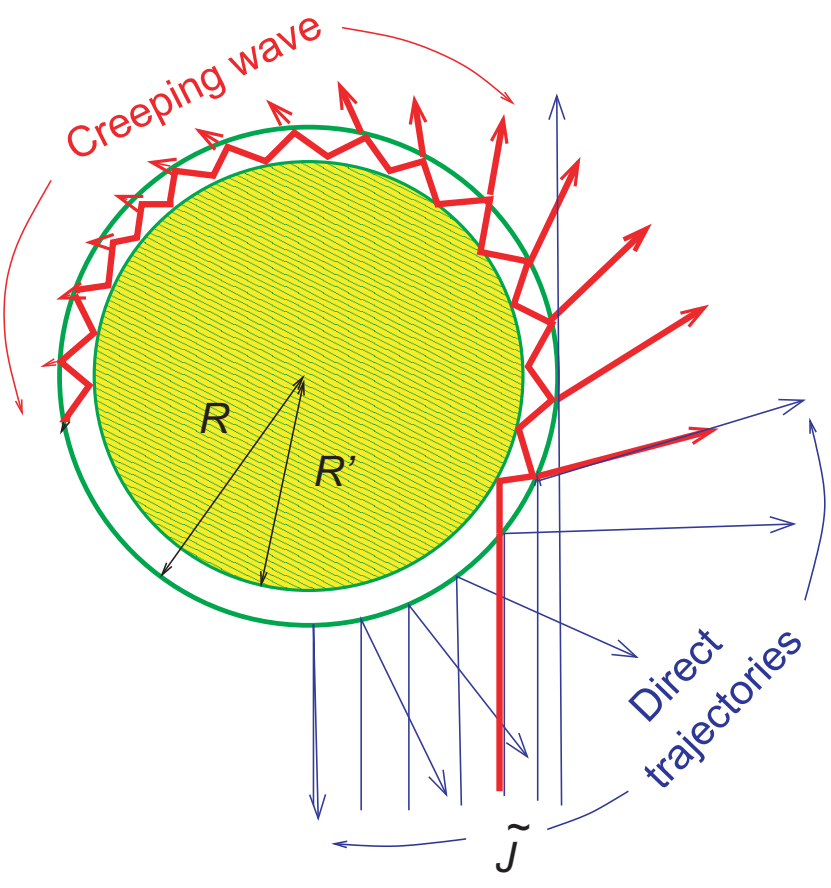

Figure 1. Particles scattered by a large hard sphere surrounded by a semi-transparent layer. The direct trajectories bounce off the outer layer (thin solid), whereas the particle with an angular momentum close to $\tilde{J}$ may become trapped between the spheres.

with $J=0$ and $E \approx E_{0}$ may penetrate the barrier and be detained between the core and the outer layer for a period of time determined by the value of $\Omega$. A particle with $J \neq 0$ and $E>E_{0}$ may also be trapped in a similar way as the centrifugal potential raises the floor of the well by $\approx J(J+1) / R^{2}$, and may align the energy of the level with that of the incident particle. In this case, a particle (ray) with an energy $E$ and the angular momentum $\tilde{J}$ such that $E_{0}+\tilde{J}(\tilde{J}+1) / R^{2}=E$, may enter the well and, since the angular momentum is conserved, would continue to orbit the core as a 'creeping wave' (Regge state) until finally escaping back to the continuum.

For our model, the $S$-matrix $S(J, E)$ is known analytically (equation (21) of [6]) and, as in [6], we will use it to construct a 'reactive' scattering matrix element $S^{\text {react }}(J, E)$ by suppressing the contributions from higher angular momenta, since a reaction is less likely to occur at large impact parameters when the collision partners pass each other at a considerable distance. We, therefore, write

$$
S^{\text {react }}(J, E)=\exp \left(-J^{2} / \Delta^{2}\right) S(J, E),
$$

where $\Delta$ is an adjustable cut-off parameter. The cut-off will also help us avoid the large diffraction peak which would otherwise occur for small scattering angles [6]. Now for an impenetrable barrier, $k \Omega \gg 1$, no creeping wave can be formed and our model reduces to the scattering off a hard sphere of radius $R$. The corresponding DCS is shown in figure 2(a) versus the energy $E$ and the scattering angle $\theta$. The DCS is practically structureless except for minor ripples at lower energies, which are all that remains of the diffraction peak. The presence of a relatively long-lived resonance state (the creeping wave) does, however, change things dramatically. The DCS for $\Omega=5$, shown in figure 2(b), 

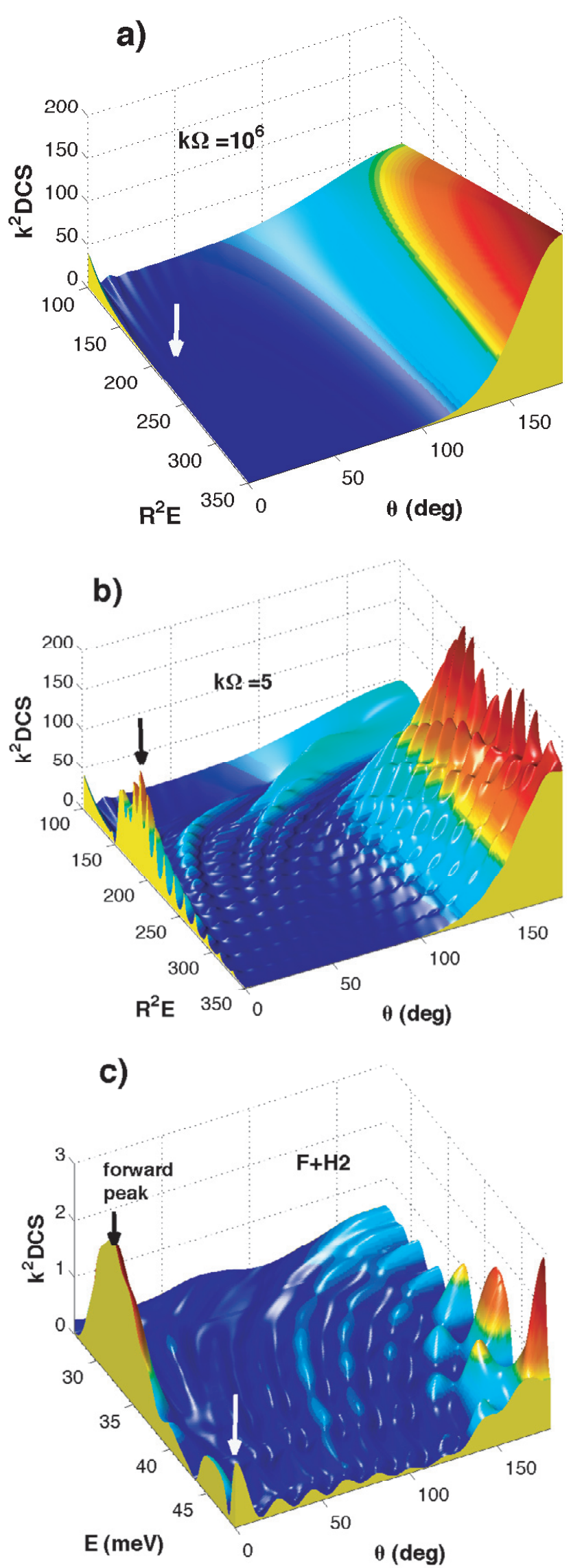

Figure 2. DCS versus scattering angle $\theta$ and collision energy $E$ for (a) the model in the figure 1 with an impenetrable outer layer, $k \Omega=10^{6}$; (b) for the same model with $k \Omega=5$; (c) for the $\mathrm{F}+\mathrm{H}_{2}(v=0, j=0) \rightarrow \mathrm{FH}\left(v^{\prime}=2, j^{\prime}=0\right)+\mathrm{H}$ reaction. The arrows indicate the energies in figures 4 (a)-(d). is highly structured and exhibits characteristic ridges and troughs bending towards the observer, as well as a pronounced forward peak for $150<R^{2} E<250$. This is the resonance effect we will analyse first.

\subsection{Regge trajectories.}

Mathematical description of the effect is based on the concept of Regge poles, i.e. poles of the $S$-matrix element, evaluated at a fixed energy $E$, in the complex $J$-plane (CAM plane). The trapping of the particle at, say, some real $J \approx \tilde{J}$ suggests that at some CAM $J_{0}=\operatorname{Re} J_{0}+\mathrm{i} \operatorname{Im} J_{0}, \operatorname{Re} J_{0} \approx \tilde{J}$ the $S$-matrix element diverges, i.e. the radial part of the regular wave function $\Psi^{J}(r)$ satisfies the boundary conditions

$$
\begin{aligned}
& \Psi^{J_{0}}(r) \rightarrow 0, \quad \text { for } r \rightarrow 0, \quad \text { and } \\
& \Psi^{J_{0}}(r) \rightarrow \exp (\mathrm{i} k r) \quad \text { for } \quad r \rightarrow \infty
\end{aligned}
$$

and does not contain an incoming wave for large values of $r$. Typically the $S$-matrix element has many poles at $J=J_{n}$, $n=0,1, \ldots$ whose positions change as the energy $E$ varies. In our simple case we are only interested in the pole at $J=J_{0}$ associated with the capture in the lowest metastable state $(E \approx$ $\left.E_{0}\right)$ supported by the potential (1). Above we have already estimated the dependence of its real part on $E$ by requiring the centrifugal potential shifts the energy of the metastable state in order to align it with the energy of the incident beam, $\operatorname{Re} J_{0}\left(\operatorname{Re} J_{0}+1\right) / 2 R^{2}+E_{0}=E$, which for $\operatorname{Re} J_{0} \gg 1$ reads

$$
\operatorname{Re} J_{0} \approx\left[\left(E-E_{0}\right) / B\right]^{1 / 2},
$$

where $B=1 / 2 R^{2}$ is the rotational constant. Similarly, a crude estimate for the dependence of $\operatorname{Im} J_{0}$ on $E$ can be obtained [7] by recalling that just as $\tau=1 / \Gamma_{0}$ gives the lifetime of a metastable state, $\vartheta \equiv 1 /\left(2 \operatorname{Im} J_{0}\right)$ gives the angular life of the Regge state, i.e. the angle by which the creeping wave would progress before finally disappearing. Estimating $\vartheta \approx \omega \tau$, where $\omega$ is the angular velocity of the trapped particle, and recalling that the kinetic energy available for rotation is $E-E_{0}$ suggests that $\operatorname{Im} J_{0}$ would decrease with energy as

$$
\operatorname{Im} J_{0} \approx \Gamma_{0}\left[\left(E-E_{0}\right) / B\right]^{-1 / 2} / 4 .
$$

Note that these estimates require $E \gg E_{0}$ and a more detailed analysis is needed for $E \approx E_{0}$. Dependence of $\operatorname{Re} J_{0}$ and $\operatorname{Im} J_{0}$ on $E$ is shown in figures 3(b) and (c), respectively, while the Regge trajectory, $\operatorname{Im} J_{0}$ versus $\operatorname{Re} J_{0}$, is plotted in figure 3(a).

Another useful, if more formal, way to look at the Regge poles is to recognize in the radial Schrödinger equation with the boundary conditions (3) an (irregular) Sturm-Liouville problem in which $\Lambda_{n} \equiv J_{n}\left(J_{n}+1\right)$ plays the role of the eigenvalue [4]. The energy $E$ enters as a parameter, and the eigenvalue $\Lambda_{n}(E)$ is a function single-valued on a Sturmian Riemann surface consisting of an infinite number of complex $E$-planes joined at the branching points. Thus, proceeding along the real $E$-axis on the $n$th sheet one reads off $\Lambda_{n}(E)$ and the position of the $n$th Regge pole, $J_{n}(E)$. In most cases the branching points lie far away from the real $E$-axis, and different Regge trajectories appear to be completely independent. For example, equations (4) and (5) amount to assuming a linear dependence $\left(\alpha_{1,2}\right.$ and $\beta$ are real constants)

$$
\Lambda_{n}(E) \approx\left(\alpha_{1}+\mathrm{i} \alpha_{2}\right)+\beta E
$$




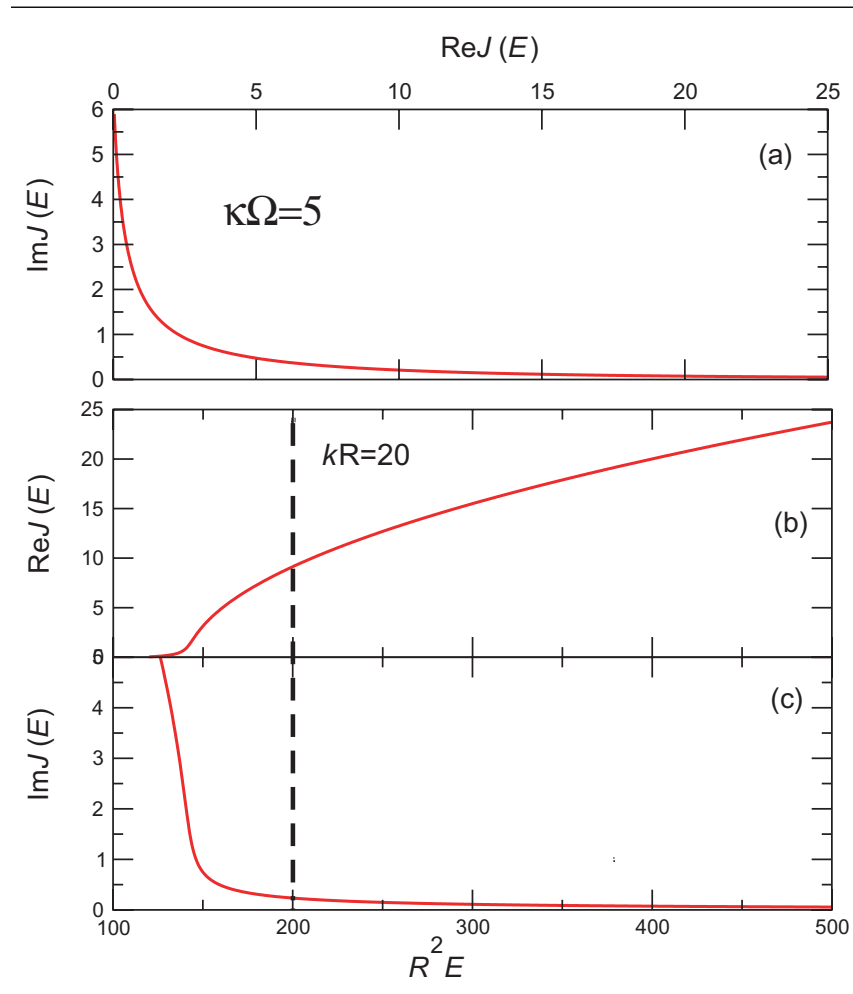

Figure 3. (a) Regge trajectory for the model in figure 1 with $k \Omega=5$; (b) the real part of the same trajectory versus energy $E$; (c) the imaginary part of the same trajectory versus $E$. The vertical dashed line indicates the energy in figures $4(a)$ and (b).

in the energy range where $\operatorname{Re} J_{0} \gg \operatorname{Im} J_{0}$. Equation (6) no longer applies close to one of the branch singularities, for example, in the presence of two metastable states which can be aligned by varying the centrifugal potential $J(J+1)$ / $\left(2 R^{2}\right)$. An example of such behaviour will be given below.

\section{2. $D C S$}

The mechanism responsible for the structure seen in figure 2(b) is easily deduced from figure 1 . Without capture in the Regge state, i.e. for $k \Omega \gg 1$, only one classical trajectory is scattered into any given angle $\theta$ (the direct contribution). With the creeping wave present the number of ways to be scattered into an angle $\theta$ increases: for example, the particle can join the creeping wave and travel with it (a bit like riding a merry-go-round) until leaving again in such a manner that its radius-vector $\vec{r}$ sweeps, in total, the angle $\Delta \varphi=\pi-\theta$ (the first nearside resonance contribution). Due to the axial symmetry of the problem, $\vec{r}$ can also rotate to sweep the angle $\Delta \varphi=\pi+\theta$ (the first farside resonance contribution). If the resonance is sufficiently long-lived, $\vec{r}$ may also complete $m$ full rotations before settling in the same final direction, thus giving rise to the $m$ th nearside or farside contribution. The pattern in figure 2(b) is a result of the interference between all the above scenarios. The standard PWS used to evaluate the scattering amplitude $\left(P_{J}(z)\right.$ denotes a Legendre polynomial)

$$
f(\theta, E)=(\mathrm{i} k)^{-1} \sum_{J=0}^{\infty}(J+1 / 2) P_{J}(\cos (\theta)) S(J, E)
$$

does not distinguish between the nearside or farside terms. We require, therefore, a different representation that would decompose $f(\theta, E)$ into the terms describing direct scattering off the outer sphere and decay of the creeping wave into the nearside and farside regions. In the semiclassical limit, it can be achieved by writing (for more detail see $[6,8]$ )

$$
\begin{aligned}
f(\theta, E) \simeq & (\mathrm{i} k)^{-1}[2 \pi \sin (\theta)]^{-1 / 2} \\
& \times \sum_{m=0}^{\infty} \tilde{f}\left(\varphi_{m}\right) \exp (-\mathrm{i} \pi / 4-\mathrm{i} m \pi / 2),
\end{aligned}
$$

where $\left.\varphi_{m}=(-1)^{m+1} \theta+\pi\left[m+1 / 2+(-1)^{m} / 2\right)\right]$ and

$$
\tilde{f}(\varphi)=\tilde{f}^{\mathrm{opt}}(\varphi)+\delta \tilde{f}^{0}(\varphi) .
$$

In equation (8), we have introduced the winding angle $-\infty<$ $\varphi<\infty$ between the initial and final direction of the particle's radius vector $\vec{r}$ and the 'unfolded' amplitude $\tilde{f}(\varphi)$ such that $f(\theta, E)$ can be found by summing $\tilde{f}(\varphi)$ over all angles $\varphi_{m}$ consistent with the observed scattering angle $\theta$. In equation (9) the unfolded amplitude $\tilde{f}(\varphi)$ is written as a sum of the direct, $\tilde{f}^{\text {opt }}(\varphi)$, and the resonance, $\delta \tilde{f}^{0}(\varphi)$, contributions, whose explicit forms are given by equations (15) and (19) of [6]. For the direct trajectories $\varphi$ does not exceed $\pi$ and, for this reason, $\tilde{f}^{\mathrm{opt}}(\varphi)$ occupies the first nearside region, $0<\varphi<\pi$. The resonance term describes the particle travelling with the creeping wave (Regge state). It rapidly rises at first, and as the creeping wave progresses around the core becomes a decaying exponential tail, which may extend well beyond the first nearside region,

$$
\delta \tilde{f}^{0}(\varphi) \sim \rho_{0} \exp \left(i \varphi \operatorname{Re} J_{0}-\varphi \operatorname{Im} J_{0}\right),
$$

where $\rho_{0}$ denotes the residue of the $S$-matrix element at $J=J_{0}$,

$$
\rho_{0} \equiv \lim _{J \rightarrow J_{0}}\left(J-J_{0}\right) S(J, E) .
$$

Note that if there are poles other than at $J_{0}$, more resonance terms $\delta \tilde{f}^{n}(\varphi)$, one for each pole, $n=1,2 \ldots$, would be added in equation (9) [6]. For the parameters chosen in figure 2(b) equations (8) and (9) reproduce to a graphical accuracy the exact scattering amplitude in equation (7) everywhere except for very small, $\theta \approx 0$, and very large, $\theta \approx \pi$ scattering angles [6] where the nearside and farside contributions become indistinguishable and the scattering amplitude (8) diverges. The semiclassical asymptotes for the small- and large-angle scattering amplitudes, which allow to extend the CAM analysis to the whole angular range $0 \leqslant \theta \leqslant \pi$, can be found in [8].

The formation of the oscillatory pattern in the DCS seen in figure 2(b) for $k R=20$ and $k \Omega=5$ is illustrated schematically in figure 4. In figure 4(a) the non-resonance DCS for $k \Omega=10^{6}$ is plotted versus the scattering angle $\theta$ measured counter-clockwise along a large semi-circle in the forefront. For a practically impenetrable barrier, the metastable state becomes a true bound state inaccessible to the incoming particles. Accordingly, the DCS has a direct backward peak at $\left(\theta \approx 180^{\circ}\right)$ and only a smooth direct contribution $\tilde{f}^{\text {opt }}(\varphi)$, which we chose to plot in the background versus $\varphi$ measured counter-clockwise. For $k \Omega=$ 5 (figure $4(\mathrm{~b})) \tilde{f}^{\text {opt }}(\varphi)$ is almost identical to the one shown in figure $4(\mathrm{a})$ as the direct trajectories continue to be reflected by the outer sphere in much the same manner. There is, 

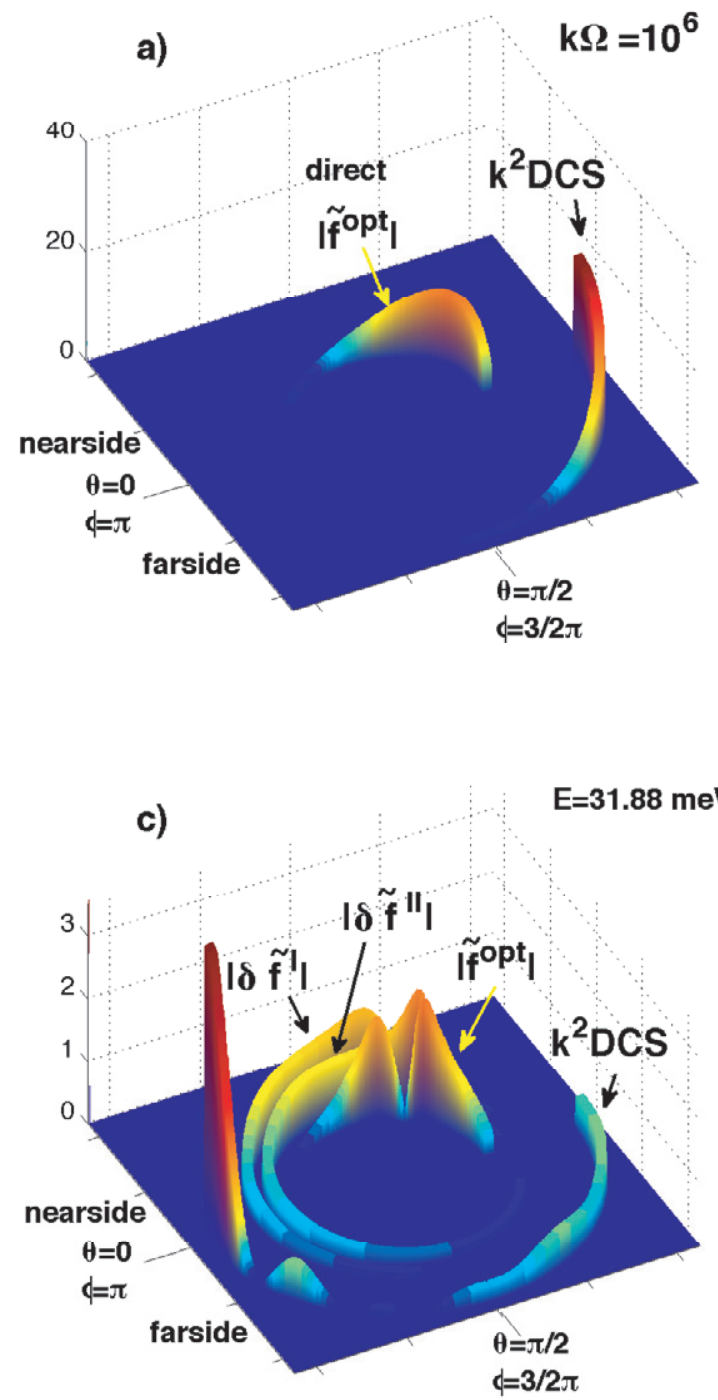
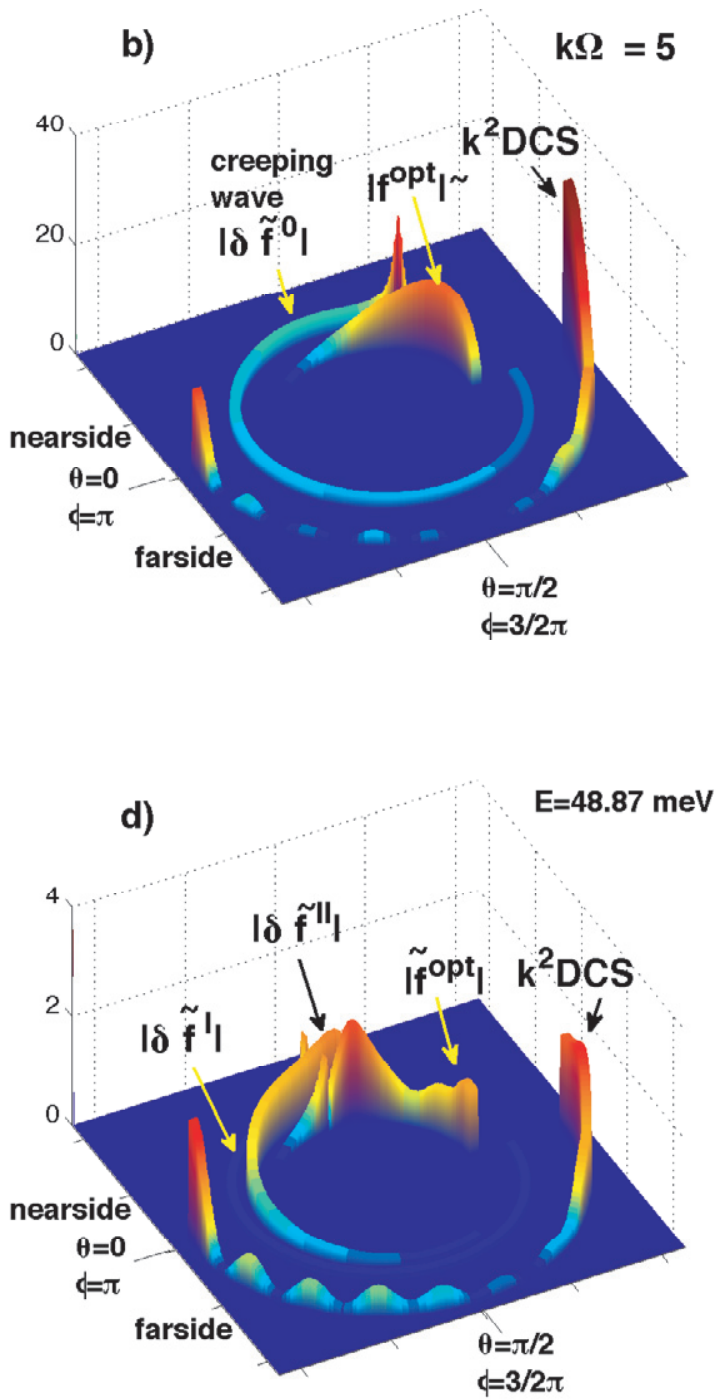

Figure 4. Decomposition of a DCS into the direct and resonance parts for: (a) the model in figure 1 with $k \Omega=10^{6}$ at $k R=20$; (b) the model in figure 1 with $k \Omega=5$ at $k R=20$; (c) for the $\mathrm{F}+\mathrm{H}_{2}(v=0, j=0) \rightarrow \mathrm{FH}\left(v^{\prime}=2, j^{\prime}=0\right)+\mathrm{H}$ reaction at $E=31.88 \mathrm{meV}$; (d) for the $\mathrm{F}+\mathrm{H}_{2}(v=0, j=0) \rightarrow \mathrm{FH}\left(v^{\prime}=2, j^{\prime}=0\right)+\mathrm{H}$ reaction at $E=48.87 \mathrm{meV}$. To allow for better viewing, the DCS were multiplied by the factor of 0.2 in (a) and (b) and by the factor of 2 in (c) and (d).

however, also a creeping wave described by the resonance term $\delta \tilde{f}^{0}$ plotted versus $\varphi$ measured counter-clockwise along the larger circle. The life angle of the resonance is about $125^{\circ}$ so that the exponential tail extends to the first farside region and then becomes negligible. The full scattering amplitude is then obtained by folding the tail onto the $[0, \pi]$ interval as prescribed by equation (7) and adding all three amplitudes. The resultant DCS shows a pronounced backward peak, a smaller forward peak and a number of smaller oscillations in between. It is readily seen that the forward peak must result from the interference between the first farside and the first nearside resonance terms. The oscillations superimposed on the direct backward peak are caused by the interference between the direct and the first farside resonance terms while the sideways minimum at $\theta \approx$ $90^{\circ}$ is produced by the destructive interference between all three contributions. Below we will demonstrate that angular distributions similar to the one shown in figure 4 occur in realistic systems whenever a single resonance contributes to a reactive transition.

\subsection{ICS.}

Next we search the model shown in figure 1 for the evidence of resonance patterns in the energy dependence of the ICS, using the original uncut ${ }^{1} S$-matrix element $S(J, E)$ in equation (2) which, of course, has the same pole structure as $S^{\text {react }}(J, E)$ employed above. The ICS for an impenetrable, $k \Omega=10^{6}$, barrier,

$$
\sigma(E)=\pi / k^{2} \sum_{J=0}^{\infty}(2 J+1)|S(J, E)-1|^{2},
$$

1 Since $\left|S^{\text {react }}(J, E)\right|^{2}=\exp \left(-J^{2} / \Delta^{2}\right)$, an ICS computed with it would be independent of energy and to obtain a structured ICS we must return to $S(J, E)$ ). 


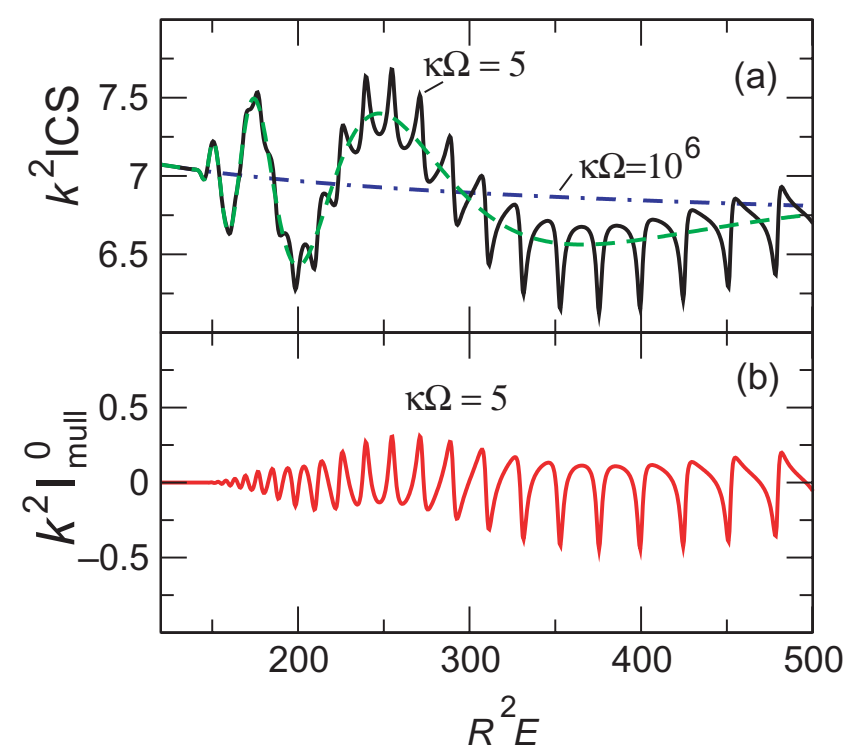

Figure 5. (a) The ICS for the model in figure 1 with $k \Omega=10^{6}$ (dot-dashed) and $k \Omega=5$ (solid). Also shown by the dashed line is the result of subtraction of the Mulholland contribution (below) from the solid curve; (b) the Mulholland contribution for $k \Omega=5$.

plotted in figure 5(a) versus $E$ by a dot-dashed line, is smooth and structureless. Again, the presence of a resonance leads to significant changes. The ICS for $k \Omega=5$ (solid) shows a pattern which begins to affect the cross-sections at, roughly, the energies when the resonance begins to influence the DCS in figure 2(b). The pattern starts as slow sinusoidal oscillations which, at higher energies, become less regular and eventually are converted into narrow downward spikes which occur each time the Regge trajectory in figure 3(a) passes near a real integer value of $J$. Just like the PWS (7), the PWS (12) is not well suited for separating contributions from resonance and direct mechanisms. A more convenient representation was recently proposed by Macek et al [9] in the form of the Mulholland formula [10]

$$
\begin{aligned}
\sigma(E)= & 4 \pi k^{-2} \int_{0}^{\infty} \operatorname{Re}[1-S(\lambda, E)] \lambda \mathrm{d} \lambda \\
& -8 \pi^{2} k^{-2} \operatorname{Im} \frac{\lambda_{0} \rho_{0}}{1+\exp \left(-2 \pi \lambda_{0}\right)}+I(E),
\end{aligned}
$$

where we have introduced the variable $\lambda=J+1 / 2, \lambda_{0}=$ $J_{0}+1 / 2$ and $\rho_{0}$ is the residue of the $S$-matrix element already defined in equation (10). The first term in equation (13) is just the result of replacing the PWS (12) with an integral while $I(E)$ contains certain integrals along the imaginary $\lambda$-axis and is expected to be small or at least smooth. The second term is the Mulholland contribution, $I_{\text {mul }}^{0}$, which we expect to be responsible for the pattern in figure 5. Note that if there are poles at other locations $J_{n} n=1,2 \ldots$, more Mulholland contributions, one for each pole, would be added to equation (13) [9].

The plot of the Mulholland contribution shown in figure 5(b) confirms that the rapid oscillations are indeed produced by the Regge trajectory shown in figure 3 . The curve obtained by subtracting the Mulholland curve from the full ICS, shown in figure 5(a) by a dashed line, is smooth apart from the three oscillations for $R^{2} E<350$, which also has a resonance origin. The reason the Mulholland formula (13) does not account for this remaining structure is simple. By construction [11], the Mulholland term contains contributions from the processes in which the resonance complex (the creeping wave) completes at least one full rotation. The slow oscillations, on the other hand come from the first round $(\varphi \leqslant 2 \pi)$ whose contribution is hidden in two other terms in equation (13). The Mulholland formula can be modified to also account for the remaining structure and leave the non-resonance contribution similar to the dot-dashed curve in figure 5. Such an extension is, however, beyond the scope of this paper and will be discussed elsewhere [12].

\section{The $\mathrm{F}+\mathrm{H}_{2}(v=0, j=0) \rightarrow \mathrm{FH}\left(v^{\prime}=2, j^{\prime}=0\right)+\mathrm{H}$ reaction}

We proceed with analysing resonance effects in the state-to-state DCS and ICS of the $\mathrm{F}+\mathrm{H}_{2} \rightarrow \mathrm{FH}+\mathrm{H}$ reaction. The zero-helicity $S$-matrix elements $S_{v^{\prime} \leftarrow v}(J, E), v \equiv(v, j)$ were computed with the help of the hyperquantization algorithm of Aquilanti et al [13] on the Stark-Werner potential surface [14] for the physical integer values of the total angular momentum $J$. The information about the behaviour of the $S$-matrix element in the complex $J$-plane, such as the values of $S_{v^{\prime} \leftarrow v}(J, E)$ for complex values of $J$, Regge poles positions and the corresponding residues, was obtained by the Padé reconstruction method, a detailed discussion of which can be found, for example, in [6]. Mathematically, our discussion will be very similar to that of the previous section, as both cases involve Regge poles associated with the resonance states trapped in their respective potential wells. The $\mathrm{F}+\mathrm{H}_{2}$ example is, however, more complex as we will have to deal with two, rather just one, interacting resonances [4]. The physical interpretation of the reactive resonance effects is also similar to the one offered in section 2, provided one replaces the picture of a particle trapped between the potential walls by that of an intermediate tri-atomic (quasi) molecule. Just as the creeping wave must propagate around the core to preserve the total angular momentum, the complex must rotate. By the time the bond(s) break, releasing the outgoing atom and leaving the product di-atomic in the specified vibrational $\left(v^{\prime}\right)$ and rotational $\left(j^{\prime}\right)$ state, this rotation may have already carried the complex into an angular range beyond the reach of the direct trajectories.

\subsection{Regge trajectories.}

In the collision energy range $25-50 \mathrm{meV}$ the $v^{\prime}=2, j^{\prime}=$ $0 \leftarrow v=0, j=0$ transition is affected by the presence of two resonances. Chao and Skodgje [15] have shown that the system has two potential wells sustaining two metastable levels, schematically shown in figure 6 . When the centrifugal potential is added, both levels move upwards, albeit at different speeds. The level in the well labelled $A$, located closer to the transition state, moves faster, aligns with the state labelled $B$ and then overtakes it. In the CAM approach we use the centrifugal potential to align the position of a metastable level $\left(E_{\mathrm{A}}\right.$ or $\left.E_{\mathrm{B}}\right)$ with the energy of the incident beam. As it happens, for $E^{\text {cross }} \approx 38 \mathrm{meV}$ and $J \approx 7$ all three energies become aligned, $E_{\mathrm{A}} \approx E_{\mathrm{B}} \approx E$, and the particles 


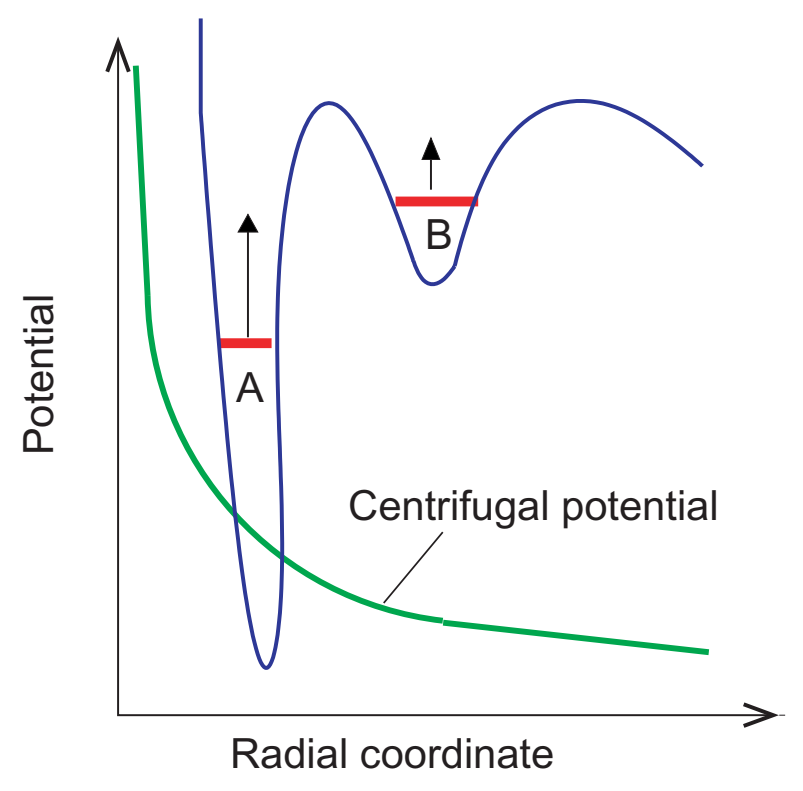

Figure 6. Schematic drawing of a double-well potential supporting two metastable levels (Feshbach resonances) A and B. As a centrifugal potential is added, both levels move up, A faster than B.

end up captured not in the state $\mathrm{A}$ or $\mathrm{B}$ but in appropriate linear combinations of the two [4]. Although $E_{\mathrm{A}} \approx E_{\mathrm{B}}$, the overlap between the two states splits the positions of the two Regge poles, which are nevertheless very close [4]. Examining the complex $E$-plane one finds, close to the real $E$-axis, the point $E^{(1)}$ where the positions of the two Regge poles coincide. Recalling that, as was discussed above, the two pole trajectories take their values from the same function $\Lambda(E)$ evaluated on different sheets of its Riemann surface, we recognize $E^{(1)}$ as a branching point at which the two Riemann sheets are joined. If the interaction $\delta$ between the states A and B were put to zero (we assume for a moment that we can do it), the two sheets would disconnect. This suggests that the branching points come in pairs and $E^{(1)}$ must have its counterpart $E^{(2)}$, which would move towards and finally coalesce with $E^{(1)}$ as $\delta \rightarrow 0$ so that the cut joining the two points will disappear. Close to $E^{(1)}$ we can no longer pretend that the two Regge trajectories are independent, and to describe them must use, instead of equation (6), a form which accounts for the presence of the two branching points [4]. It is convenient to write the equations as

$$
\operatorname{det}\left(\begin{array}{cc}
\alpha_{\mathrm{I}}+\beta_{\mathrm{I}} E-\Lambda, & \delta \\
\delta, & \alpha_{\mathrm{II}}+\beta_{\mathrm{II}} E-\Lambda
\end{array}\right)=0 .
$$

where $\Lambda \equiv J(J+1)$ and the (complex) values of $\alpha_{i}, \beta_{i}$ and $\delta$ need to be determined numerically. The two Regge trajectories labelled (I) and (II) are shown in figure 7(a) (circles) and the energy dependence of their real and imaginary parts is shown in figures 7(b) and (c) (circles). In all three plots the solid lines show the result of fitting the pole positions to the form (14). Note that the imaginary parts of the two trajectories cross, while their real parts exhibit an avoided crossing, which indicates that the two branching points are located on opposite sides of the real $E$-axis [4]. Note also that there is no a priori reason why that should be the case: the two branching points can, in principle, lie on the same side of the real axis, causing the real rather than the imaginary

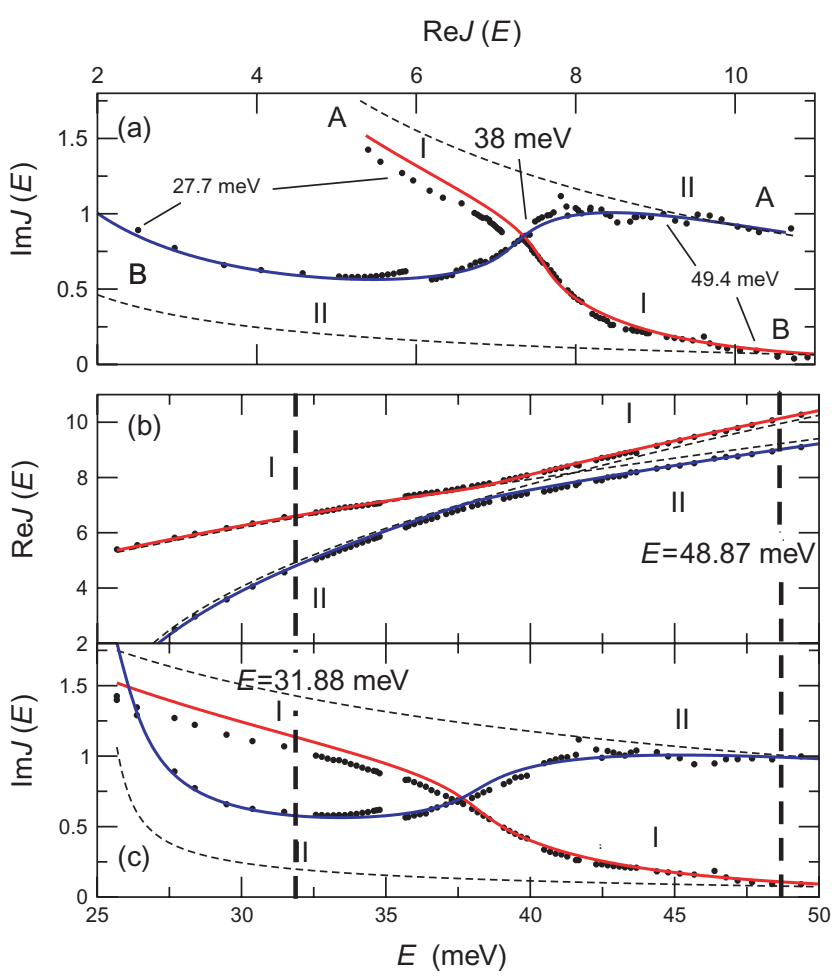

Figure 7. (a) Regge trajectories for the $\mathrm{F}+\mathrm{H}_{2}(v=0, j=0) \rightarrow$ $\mathrm{FH}\left(v^{\prime}=2, j^{\prime}=0\right)+\mathrm{H}$ reaction; (b) the real parts of the same trajectories versus energy $E$; (c) the imaginary parts of the same trajectories versus $E$. Thin dashed lines show the behaviour of the Regge trajectories if interaction between the metastable states is switched off by putting $\delta=0$ in equation (14). Vertical dashed lines indicate the energies in figures $4(\mathrm{c})$ and (d).

parts of the Regge trajectories to cross. In the rare case of a branching point $E^{(1)}$ lying on the real $E$-axis, both parts of the two trajectories would cross, $J_{\mathrm{I}}\left(E^{(1)}\right)=J_{\mathrm{II}}\left(E^{(1)}\right)$.

\subsection{DCS}

The DCS for the $v^{\prime}=2, j^{\prime}=0 \leftarrow v=0, j=0$ transition shown in figure 2(c) is remarkably similar to the model angular distribution shown in figure 2(b). It exhibits a similar pattern of ridges and troughs although is, arguably, more structured, as one would expect in the presence of two interacting resonances. The main difference is the much more pronounced forward peak centred at $E \approx 32 \mathrm{meV}$ recently observed in a beam experiment [1]. The decomposition of the DCS at the collision energy $E=31.88 \mathrm{meV}$ is shown in figure $4(\mathrm{c})$. The direct contribution $\tilde{f}^{\text {opt }}(\varphi)$ contributes mostly to the large scattering angles and is cleaved down the middle, owing to the proximity of complex (Regge) zero [16] of the $S$-matrix element. In addition, figure 4(c) exhibits two exponential tails of comparable magnitude, $\delta \tilde{f}^{\mathrm{I}}(\varphi)$ and $\delta \tilde{f}^{\mathrm{II}}(\varphi)$, which extend into the first farside region. Figure 4(c) clearly shows that the large forward peak, which occurs about $6 \mathrm{meV}$ below the energy at which the states A and B become aligned, is produced by constructive interference between the small angle decay of the corresponding Regge states. Indeed, as one might expect (see discussion following equation (2)), direct scattering into small scattering angles requires large angular momenta (impact parameters) which do not contribute to the reaction. Thus, the forward direction can be reached by 


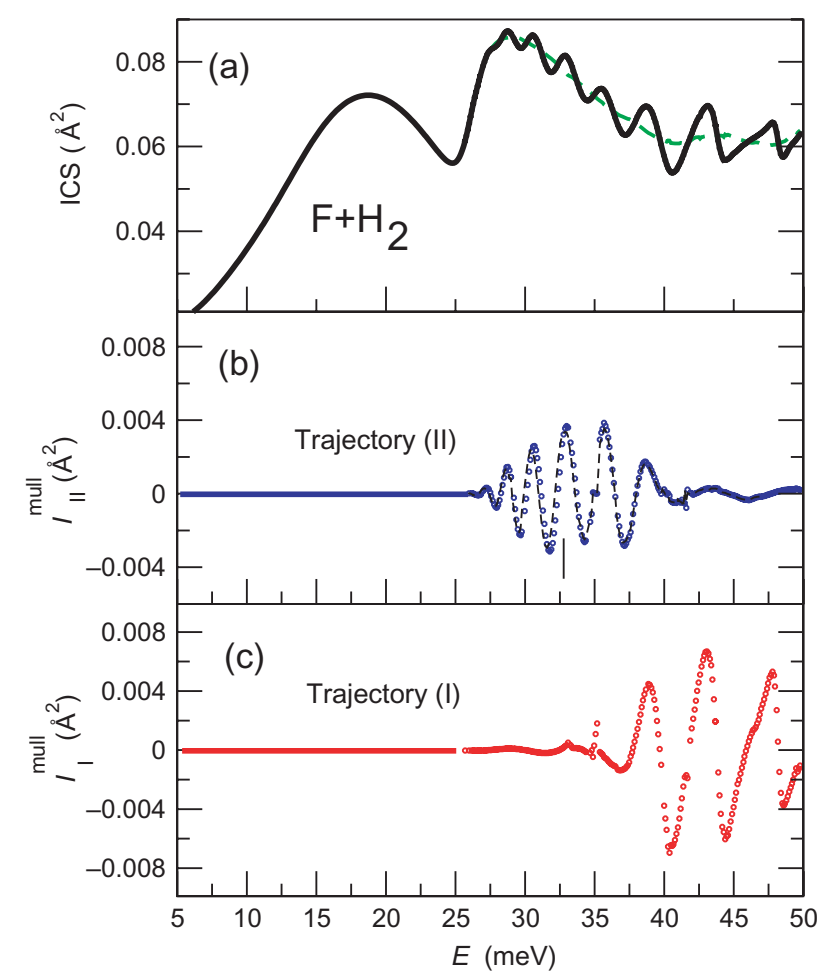

Figure 8. (a) The ICS for the $\mathrm{F}+\mathrm{H}_{2}(v=0, j=0) \rightarrow \mathrm{FH}\left(v^{\prime}=2\right.$, $\left.j^{\prime}=0\right)+\mathrm{H}$ reaction. Also shown by the dashed line is the result of subtraction of the Mulholland contributions (below) from the ICS; (b) the Mulholland contribution from the Regge trajectory (II) in figure 7; (c) the Mulholland contribution from the Regge trajectory (I) in figure 7.

the outgoing atom only by forming first a long-lived complex and travelling with it until leaving with $\theta=0$. There are two such complexes, associated with the Regge poles (I) and (II), and two interfering resonance pathways to $\theta=0$, which may either cancel or amplify each other. This conclusion was confirmed by the rigorous analysis of Sokolovski et al [8].

At a higher energy, $E \approx 48.87 \mathrm{meV}$, the DCS (seen as the face of the plot in figure 2(c)) becomes very similar to that for our model shown in figure 4(b). Its decomposition shown in figure 4(d) offers a similar explanation: at this energy only one of the two poles, namely (II) with the life angle of $\vartheta \approx 90^{\circ}$ continues to contribute to the state-to-state DCS. The pole (I), on the other hand, is on its way to become, at yet higher energies, detached from the reactants continuum $[4,15]$. Although its life angle has increased to about $280^{\circ}$, the residue of the $S$-matrix element is dramatically reduced by the proximity of the Regge zero seen in figure 4(d) as a sharp narrow minimum in the direct term $\tilde{f}^{\text {opt }}(\varphi)$. The exponential tail produced by $\delta \tilde{f}^{\mathrm{I}}(\varphi)$ is barely visible in figure $4(\mathrm{~d})$ and can be neglected. As one would expect the resultant DCS has a shape similar to that for a hard-sphere model of section 2 .

\subsection{ICS.}

The state-to-state ICS $v^{\prime}=2, j^{\prime}=0 \leftarrow v=0, j=0$ shown in figure 8(a) also bears a close resemblance to the model ICS in figure 5(a). It shows slow oscillations followed by smaller oscillations of a higher frequency which we expect to be caused by one or both Regge trajectories shown in figure 7. In its original form (13) the Mulholland formula applies to potential scattering and implicitly uses the fact that for a real angular momentum the modulus of the scattering matrix element is unity, $|S(\lambda, E)|=1$ for $\operatorname{Im} \lambda=0$. This is no longer true for a system with several open channels, and in [17] it was shown that to extend the formula to inelastic and reactive transitions one needs to replace $\left({ }^{*}\right.$ denotes complex conjugation)

$$
\begin{aligned}
& 4 \operatorname{Re}[1-S(\lambda, E)] \rightarrow\left|S_{v^{\prime} \leftarrow v}(\lambda, E)\right|^{2} \text { and } \\
& 2 \rho_{n} \rightarrow \rho_{n} S_{v^{\prime} \leftarrow v}^{*}\left(\lambda_{n}^{*}, E\right)
\end{aligned}
$$

in the integral term and the Mulholland contributions of equation (13), respectively. Plotting the Mulholland contributions for the two Regge trajectories (figures 8(b) and (c)) shows that the oscillatory pattern in figure 8(a) is indeed a resonance effect. The trajectory (II) contributes roughly to the first half of the pattern for $25 \mathrm{meV}<$ $E<40 \mathrm{meV}$, while the trajectory (I) is responsible for its other half at $35 \mathrm{meV}<E<50 \mathrm{meV}$ and there is an energy range $35 \mathrm{meV}<E<40 \mathrm{meV}$ where both contributions are considerable. To describe scattering in physical terms, we note that following the Regge trajectory (I) from lower to higher energies we start in the state A but end up in the state B, while a similar journey along the trajectory (II) takes us from B to A. Indeed, if we switch off the interaction between the metastable states, (something we can now easily do by putting $\delta=0$ in equation (14)), the state A on its way up will simply pass by the state $\mathrm{B}$, so that the real parts of the trajectories will cross, and the imaginary parts will remain roughly the same (dashed lines in figures 7). This allows us to identify the upper and lower ends of the Regge trajectories in figure 7(a) with the states $\mathrm{A}$ and $\mathrm{B}$, respectively. We have also seen that it is always the trajectory with the smaller imaginary part which produces the pattern in the ICS. Thus we conclude that both at the lower and higher energies the pattern is produced by capture in the state B earlier identified [17] as located in the van der Waals well of the product channel. In contrast, at the energies close to $E^{\text {cross }} \approx 38 \mathrm{meV}$, the state B mixes with the state A and there we observe rotational decay of two Regge states, given by the appropriate linear combinations of the A and B states. Finally we note that, just as in figure 5, the Mulholland formula does not account for the first maximum and the subsequent minimum in the $\mathrm{F}+\mathrm{H}_{2} \mathrm{ICS}$, which we also expect to be caused by the resonance(s). We defer a detailed discussion of whether or not this is the case to our future work [14].

\section{Conclusions}

To summarize, we hope to have provided a convincing argument in favour of the CAM methodology. The knowledge of the Regge poles position and residues allows us to represent the scattering amplitude as a result of interference between the direct and one or more resonance mechanisms. In a similar way, we are able to relate the structure in integral cross sections to one or several Regge trajectories. The CAM analysis is quantitative: not only does it relate certain features in a DCS or an ICS to the presence of resonance poles, but also provides explicit expressions for the corresponding resonance contributions. Although reactive scattering codes do not normally provide the information 
about either CE or CAM pole positions and residues, such information can be extracted from the numerical data (e.g. by the Padé reconstruction [8]) with an accuracy sufficient for describing phenomena as subtle as presudo-crossing of Regge trajectories which accompanies rotational alignment of metastable states. We note again, that the CE poles are best used where one needs to integrate over the particle's energy, e.g. for scattering of wavepacket with a fixed angular momentum, and need to be converted back to the CAM plane for analysing the DCS or ICS at a fixed energy. An example of such a conversion was given, for example, in [18] for the case of a linear dependence of $\Lambda_{n}$ on $E$, similar to that in equation (6). Finally, the possibility of converting one type of resonance pole into the other provides an independent check of both the accuracy of our Padé reconstruction and the validity of equation (14). In particular, using equation (14) to express $E$ as a function of $J$ we recover, as we must, the $J$ dependence of the energies and width of the CE resonance poles shown in figure 1 of [3] (see also figure 1 of [4]).

\section{Acknowledgments}

I am grateful to $\mathrm{V}$ Aquilanti, $\mathrm{D}$ DeFazio and $\mathrm{S}$ Cavalli for providing the numerical values of the $\mathrm{F}+\mathrm{H}_{2}(v=0, j=$ $0) \rightarrow \mathrm{HF}\left(v^{\prime}=2, j^{\prime}=0\right)+\mathrm{H}$ scattering matrix element and for useful discussions of the manuscript.

\section{References}

[1] Qui M et al 2006 Science 311144

[2] Smith F T 1960 Phys. Rev. A 118349

[3] Cavalli S and De Fazio D 2007 Phys. Scr. 76 C21

[4] Sokolovski D, Sen K S, Aquilanti V, Cavalli S and De Fazio D 2007 J. Chem. Phys. 12684305

[5] Connor J N L 1990 J. Chem. Soc. Faraday Trans. 861627

[6] Sokolovski D and Msezane A Z 2004 Phys. Rev. A 70 032710

[7] Sokolovski D and Castillo J F 2000 Phys. Chem. Chem. Phys. 224702

[8] Sokolovski D, De Fazio D, Cavalli S and Aquilanti V 2007 Phys. Chem. Chem. Phys. 91

[9] Macek J H, Krstic' P S and Ovchinnikov S Yu 2004 Phys. Rev. Lett. 93183203

[10] Mulholland H P 1928 Proc. Cambridge Phil. Soc. 24280

[11] Sokolovski D, Felfli Z, Ovchinnikov S Yu, Macek J H and Msezane A Z 2007 Phys. Rev. A 76012705

[12] Sokolovski D unpublished

[13] Aquilanti V, Cavalli S, Simoni A, Aguilar A, Lucas J M and De Fazio D 2004 J. Chem. Phys. 12111675

[14] Stark K and Werner H-J 1996 J. Chem. Phys. 1046515

[15] Chao S D and Skodje R T 2000 J. Chem. Phys. 1133487

[16] Sokolovski D 2000 Phys. Rev. A 62024702

[17] Sokolovski D, De Fazio D, Cavalli S and Aquilanti V 2007 J. Chem. Phys. 126121101

[18] Connor J N L 1972 Mol. Phys. 23717

Sokolovski D, Connor J N L and Schatz G C 1995 J. Chem. Phys. 1035979 